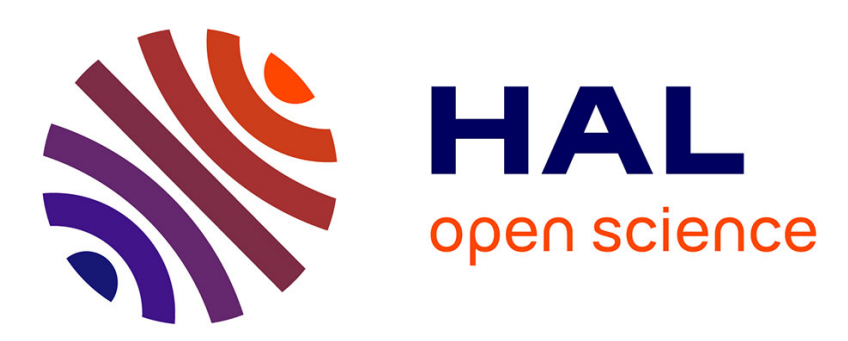

\title{
Inverse problem characterization using an adaptive database
}

\author{
Sandór Bilicz, Marc Lambert, Szabolcs Gyimóthy
}

\section{To cite this version:}

Sandór Bilicz, Marc Lambert, Szabolcs Gyimóthy. Inverse problem characterization using an adaptive database. URSI International Symposium on Electromagnetic Theory (EMT-S 2010), Aug 2010, Berlin, Germany. pp.509-512, 10.1109/URSI-EMTS.2010.5637281 . hal-00490005

\section{HAL Id: hal-00490005 https://hal.science/hal-00490005}

Submitted on 7 Jun 2010

HAL is a multi-disciplinary open access archive for the deposit and dissemination of scientific research documents, whether they are published or not. The documents may come from teaching and research institutions in France or abroad, or from public or private research centers.
L'archive ouverte pluridisciplinaire HAL, est destinée au dépôt et à la diffusion de documents scientifiques de niveau recherche, publiés ou non, émanant des établissements d'enseignement et de recherche français ou étrangers, des laboratoires publics ou privés. 


\title{
Inverse problem characterization using an adaptive database
}

\author{
Sándor Bilicz ${ }^{\#, * 1}$, Marc Lambert ${ }^{* 2}$, Szabolcs Gyimóthy \#3 \\ \# Budapest University of Technology and Economics, \\ Egry J. u. 18, H-1521 Budapest, Hungary \\ ${ }^{1}$ biliczerevt.bme.hu \\ ${ }^{3}$ gyimothy@evt.bme.hu \\ * Département de Recherche en Électromagnétisme, \\ Laboratoire des Signaux et Systèmes UMR8506 (CNRS-SUPELEC-Univ Paris-Sud), \\ 91192 Gif-sur-Yvette cedex, France \\ ${ }^{2}$ marc. lambertalss. supelec.fr
}

\begin{abstract}
In this paper, the characterization of electromagnetic inverse problems is addressed. As it is well known, an inverse problem can be ill-posed, i.e., its solution is not necessarily unique and might be quite sensitive to the measured data. To characterize such an inverse problem a combination of a surrogate model —based on an optimal database — and an inverse mapping of some sort, both using kriging prediction as tools, is proposed. The database (a kind of discrete representation of the electromagnetic model) is generated using an adaptive sampling strategy aiming to find a set of optimal input parameter-output data pairs. Once the latter has been computed, both qualitative and quantitative conclusions about the related inverse problem can be drawn.
\end{abstract}

The illustrative examples are drawn from eddy-current nondestructive testing.

\section{INTRODUCTION}

Nowadays more and more emphasis is being put on the surrogate modeling of electromagnetic phenomena [1]. The main goal of surrogate modeling is to reduce the computational burden imposed by the precise simulation of numerical models. A natural manner is to store pre-computed results in a database and to fit an interpolator to these samples. To improve the quality of the surrogate model, the choice of the samples should obviously be adapted to the problem. To achieve an "optimal" sampling (in a certain sense), several methods have been proposed. Mesh-based strategies (inspired by the mesh of Finite Element Methods) are discussed in [2] and [3]. A meshless method is proposed in [4] -this approach is recast and extended in [5], [6].

Such databases (being adapted to the problem) makes possible not only the fast approximation of a complex model, but the behavior of the underlying physical problem can also be studied: the structure of the databases bears valuable metainformation about the model. In this paper, we present a sampling strategy for the generation of adaptive databases and propose methods to make use the available meta-information in the characterization of the related inverse problem.

\section{GENERATION OF ADAPTIVE DATABASES}

The method is formalized in a general manner. Let us define a well-behaved forward operator $\mathcal{F}$ representing a forward problem with a unique solution. This makes the connection between the input vector $\mathbf{x}=\left[x_{1}, x_{2}, \ldots, x_{p}\right]^{\top}$ consisting of $p$ real parameters, and the output scalar function $y(t)$ :

$$
y(t)=\mathcal{F}\{\mathbf{x}\}, \quad t \in T \subset \mathbb{R} .
$$

Let us assume that each $x_{k}(k=1,2, \ldots, p)$ input parameter has a lower and an upper bound, so the input space $\mathbb{X}$ is a $p$ dimensional hyper-cuboid. The output space is defined via the forward operator, as the codomain of $\mathcal{F}\{\mathbf{x}\}$ :

$$
\mathbb{Y}=\{y(t): y(t)=\mathcal{F}\{\mathbf{x}\}, \forall \mathbf{x} \in \mathbb{X}\} .
$$

Both $\mathbb{X}$ and $\mathbb{Y}$ are equipped with a norm: $\|\cdot\|_{x}$ and $\|\cdot\|_{y}$, respectively.

Let us define a database $\mathbb{D}_{n}$ as a set of $n$ samples, each of them being a pair of input sample $\left(\mathbf{x}_{i}\right)$ and output sample $\left(y_{i}(t)=\mathcal{F}\left\{\mathbf{x}_{i}\right\}\right), \forall i \in N . N$ is the index set of the database: $N=\{1,2, \ldots, n\}$. Formally:

$$
\mathbb{D}_{n}=\left\{\left(\mathbf{x}_{1}, y_{1}(t)\right),\left(\mathbf{x}_{2}, y_{2}(t)\right), \ldots,\left(\mathbf{x}_{n}, y_{n}(t)\right)\right\} \text {. }
$$

The main idea of our sampling strategy is to build an “optimal" database $\mathbb{D}_{n}$ such as its output samples $y_{i}(t), i \in N$ are evenly spread all over the output space $\mathbb{Y}$. By introducing the distance function $Q_{i}(\mathbf{x})$ :

$$
Q_{i}(\mathbf{x})=\left\|\mathcal{F}\{\mathbf{x}\}-y_{i}(t)\right\|_{y}, \mathbf{x} \in \mathbb{X}, \forall i \in N,
$$

the criteria of optimality are formalized as

$$
\max _{i \in N}\left[\min _{j \in N \backslash\{i\}} Q_{i}\left(\mathbf{x}_{j}\right)\right]=\min _{i \in N}\left[\min _{j \in N \backslash\{i\}} Q_{i}\left(\mathbf{x}_{j}\right)\right]
$$

and

$$
\max _{\mathbf{x} \in \mathbb{X}}\left[\min _{i \in N} Q_{i}(\mathbf{x})\right] \leq \Delta,
$$

where $\Delta$ is a parameter to be chosen, according to the sought "resolution" (depending on the user's needs). The criterion 
(5) expresses the equi-spacing of output samples, whereas (6) imposes that the output space $\mathbb{Y}$ is sampled everywhere, i.e., no unexplored region can be found.

A similar idea of such an "output space filling" is introduced in [4] and referred as "response diversity". However, [4] focuses only on the criterion (6), moreover, a functional output is not considered.

\section{A. Iterative-incremental sampling strategy}

The generation of an optimal database is recast as an optimization problem which can be solved iteratively. Starting with an initial database consisting of one sample $\mathbb{D}_{1}$, the iterative "addition" procedure can be described as follows:

a) find $\mathbf{x}_{n+1}$ the most remote point from all points already present in $\mathbb{D}_{n}$ in terms of the distance function (4) by solving

$$
\mathbf{x}_{n+1}=\arg \max _{\mathbf{x} \in \mathbb{X}}\left[\min _{i \in N} Q_{i}(\mathbf{x})\right]
$$

b) compute the output $y_{n+1}(t)=\mathcal{F}\left\{\mathbf{x}_{n+1}\right\}$

c) insert the sample $\left(\mathbf{x}_{n+1}, y_{n+1}(t)\right)$ in $\mathbb{D}_{n}$ and increase $n=$ $n+1$

d) repeat steps a) to c) until the criterion (6) is met.

However, even if such a procedure yields a database satisfying (6), the equi-spacing of samples -criterion (5)- is not necessarily fulfilled. So, a "removal strategy" is introduced, removing one of the two closest samples in the output space.

The whole procedure is defined as an alternating use of two successive "addition" and one "removal" steps. Heuristically, we might expect that such a procedure converges to the "optimal" database, but no formal proof can be proposed, however. The stopping criterion to obtain an "optimal" database can be related to the expression (5) and/or (6). In our examples, a simple upper limit for $n$ is used as stopping rule.

\section{B. Realization by kriging}

The solution of the optimization problem (7) involves a quite high computational cost since the evaluation of the distance functions $Q_{i}(\mathbf{x})$ needs the solution of a forward problem. To reduce this cost, not the exact functions $Q_{i}(\mathbf{x})$ but their kriging predictions $\hat{Q}_{i}(\mathbf{x})(i=1,2, \ldots, n)$ are used. Kriging is not discussed in details herein (see e.g., [7] for an exhaustive overview or [8] as a recent review), we just mention that it computes a linear prediction $\hat{Q}(\mathbf{x})$ of an unknown scalar function $Q(\mathbf{x})$ based on some of its samples $Q\left(\mathbf{x}_{1}\right), Q\left(\mathbf{x}_{2}\right), \ldots, Q\left(\mathbf{x}_{m}\right)$ :

$$
\hat{Q}(\mathbf{x})=\sum_{j=1}^{m} \lambda_{j}(\mathbf{x}) Q\left(\mathbf{x}_{j}\right) .
$$

The coefficients $\lambda_{j}(\mathbf{x})$ are computed in a stochastic framework, as the main idea of kriging is to model the unknown function by a Gaussian random process (GP). The same coefficients $\lambda_{j}(\mathbf{x})$ can be used to predict each distance functions since the modeling GP is the same. By replacing the prediction of all distance functions according to (8) into the original optimization problem (7), the resulted simpler problem can be solved even by performing an exhaustive search on a fine grid or a Latin hypercube point-set in $\mathbb{X}$.

\section{INVERSE PROBLEM CHARACTERIZATION}

Such "quasi-optimal" databases provide some metainformation about the involved forward operator $\mathcal{F}$ as well:

a) $\mathcal{F}$ must vary rapidly within the regions of $\mathbb{X}$ where the distribution of input samples is dense, whereas a sparsely sampled region of $\mathbb{X}$ signals the flatness of $\mathcal{F}$

b) any unknown output $y(t) \in \mathbb{Y}$ can be found not farther than $\Delta$ from one of the already computed output samples $y_{i}(t)$ of $\mathbb{D}_{n}$.

To draw quantitative conclusions concerning the point a), i.e., the sensitivity of $\mathcal{F}$, two approaches are proposed in the following.

\section{A. Inverse mapping of "noise cells"}

The measured output $\tilde{y}(t)$ is always corrupted by noise, due to either the inaccuracy of the measurements, the uncertainty of the modeling assumptions, or the numerical inaccuracy of the applied forward simulator. Let us assume that a uniform noise level $\delta$ affects all measurements. For a given measured output $\tilde{y}(t)$, one has to consider all outputs $y(t)$ satisfying $\|y(t)-\tilde{y}(t)\|_{y} \leq \delta$ as possible real (noise-free) data.

For instance, by using a database $\mathbb{D}_{n}$, the sub-region $\Phi_{i}^{\delta}$ of $\mathbb{X}$ consisting of possible inputs $\mathbf{x}$ such as their corresponding outputs are closer to $y_{i}(t)$ than $\delta$ can easily be assigned. Formally, let us define the set $\Phi_{i}^{\delta} \in \mathbb{X}$ so that

$$
\begin{aligned}
\Phi_{i}^{\delta} & =\left\{\mathbf{x} \in \mathbb{X}:\left\|\mathcal{F}\{\mathbf{x}\}-y_{i}(t)\right\|_{y} \leq \delta\right\} \\
& =\left\{\mathbf{x} \in \mathbb{X}: Q_{i}(\mathbf{x}) \leq \delta\right\}
\end{aligned}
$$

This is the inverse mapping of a "noise cell" of level $\delta$, a sub-region of $\mathbb{Y}$ is projected back onto a sub-region of $\mathbb{X}$. The shape and the dimensions of the different cells $\Phi_{i}^{\delta}$ show how the noise influences the uncertainty of the solution of the inverse problem. This formalization gives an explicit expression of the attainable precision at a given noise level.

In the realization, due to the high computational cost, not the exact distance functions but their kriging predictions are used in (9). Thus, the approximate noise-cells $\hat{\Phi}_{i}^{\delta}$ can be determined.

\section{B. Inverse mapping of Voronoi cells}

Let us define $\Omega_{i}$ the output Voronoi cell related to the $i$-th output sample $y_{i}(t)$ of $\mathbb{D}_{n}$ as

$$
\Omega_{i}=\left\{y(t) \in \mathbb{Y}: i=\arg \min _{j \in N}\left\|y(t)-y_{j}(t)\right\|_{y}\right\},
$$

i.e., the set of outputs being closer to the $i$-th output sample than to any other output sample. The inverse mapping of $\Omega_{i}$ for each $i \in N$ to the corresponding subset $\Psi_{i}$ of the input space 
$\mathbb{X}$ can then be done using the distance functions. Formally, let $\Psi_{i}$ be a subset of $\mathbb{X}$, so that

$$
\begin{aligned}
\Psi_{i} & =\left\{\mathbf{x} \in \mathbb{X}: \mathcal{F}\{\mathbf{x}\} \in \Omega_{i}\right\} \\
& =\left\{\mathbf{x} \in \mathbb{X}: i=\arg \min _{j \in N} Q_{j}(\mathbf{x})\right\} .
\end{aligned}
$$

This inverse mapping can be very effective for the characterization of the inverse problem. Provided that the database $\mathbb{D}_{n}$ is approximately output-equidistant and the distance between the output samples is roughly $2 \delta$, the dimensions of $\Psi_{i}$ represent the attainable precision of the solution in each region of $\mathbb{X}$.

Here again the kriging prediction of $Q_{i}(\mathbf{x})$ is used in (11) to reduce the computational burden, so the approximate images $\hat{\Psi}_{i}$ are computed.

Quantitative meta-information can then be provided by the knowledge of $\hat{\Psi}_{i}$ via the estimation of their dimensions. To some extent, it gives the attainable precision in each input parameter. Let us define $\Delta \mathbf{x}_{i}$ as the vector of the maximum distance between the $i$-th input sample $\mathbf{x}_{i}$ and all the possible $\mathbf{x} \in \hat{\Psi}_{i}$, defined as $\Delta \mathbf{x}_{i}=\left[\Delta x_{i, 1}, \Delta x_{i, 2}, \ldots, \Delta x_{i, p}\right]^{\top}$, where

$$
\Delta x_{i, k}=\max _{\mathbf{x} \in \hat{\Psi}_{i}}\left|x_{k}-x_{i, k}\right| .
$$

$x_{k}$ and $x_{i, k}$ is the $k$-th component (parameter) of the inputs $\mathbf{x}$ and $\mathbf{x}_{i}$, respectively. The components of $\Delta \mathbf{x}_{i}$ are related to the confidence in the solution of the inverse problem.

\section{ILLUSTRATIVE EXAMPLES}

The illustrative examples are chosen from the domain of Eddy-Current Testing (ECT), a technique for revealing and characterizing material flaws within conductive specimens. Eddy-currents are generated within the examined specimen by exciting with alternating magnetic field. The magnetic field due to the eddy-currents is measured, and, might vary near the damaged zones.

In our examples, a simple ECT setup is considered (Fig. 1). An infinitesimally thin crack affects a non-magnetic, homogeneous metal plate with an electrical conductivity $\sigma=10^{6} \mathrm{~S} / \mathrm{m}$. The thickness of the plate is $d=1.25 \mathrm{~mm}$, the other dimensions are assumed to be infinite. An air-cored probe coil (driven by a time-harmonic current of frequency $f=150 \mathrm{kHz}$ ) is scanning a centered rectangular surface of $5 \mathrm{~mm} \times 20 \mathrm{~mm}$ above the damaged zone. The impedance change of the coil (influenced by the crack) is measured at $11 \times 41$ regularly spaced positions along $\alpha$ and $\beta$. The output signal $y(t)$ is then the impedance of the coil, $t$ being related to the position of the coil. The EM phenomenon is modeled using a surface integral approach. The numerical simulation -representing $\mathcal{F}$ is based on the Method-of-Moments (for details, see [9]).

The crack is perpendicular to the surface of the plate and the configuration is described by three parameters: $L$ and $D$ are the length and the depth (in \% of $d$ ) of the crack, respectively; $A$ is the co-ordinate of the center of the crack along $\alpha$. Our approach is illustrated using two configurations. In the $2 \mathrm{D}$ example only two parameters ( $L$ and $D$ ) are varying, $A$ is 0 ; in the $3 \mathrm{D}$ example, the $x$ co-ordinate of the center of the crack $(A)$ is added as input parameter. The minimum and the maximum values of each parameter are defined in Table I.

The criterion of sample insertion (7) -approximated by using (8) - is evaluated by performing an exhaustive search in $\mathbb{X}$. In the $2 \mathrm{D}$ example, a fine regular grid of $100 \times 100=$ 10000 nodes is used, whereas in the 3D example, a set of $22^{3}=10648$ points is generated by Latin hypercube sampling. The initial sample in the database $\mathbb{D}_{1}$ corresponds to the largest crack in both examples. In the $3 \mathrm{D}$ case, its position is $A=1.5 \mathrm{~mm}$. The stopping criterion is simply an upper limit for $n$.

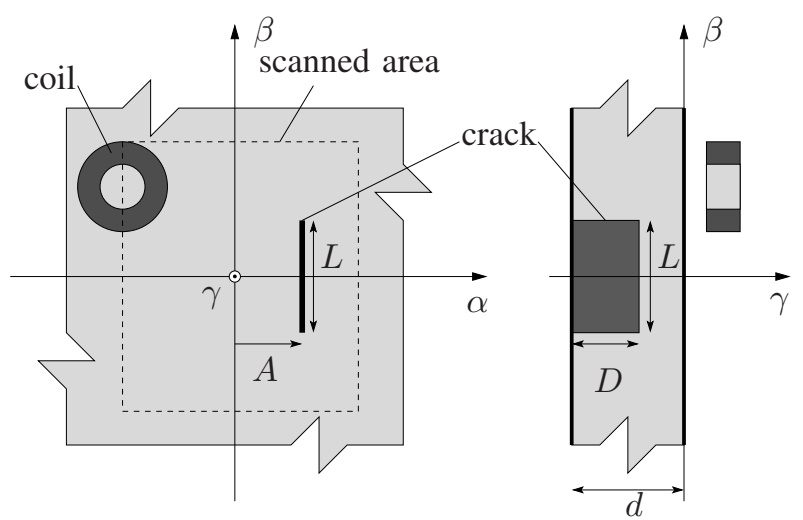

Fig. 1: Sketch of the configuration: cross-sections in the $\alpha \beta$

\begin{tabular}{|c|c|c|c|c|c|c|}
\hline \multirow{2}{*}{ Ex. } & \multicolumn{2}{|c|}{$A(\mathrm{~mm})$} & \multicolumn{2}{|c|}{$L(\mathrm{~mm})$} & \multicolumn{2}{|c|}{$D(\%)$} \\
\hline & $\min$ & $\max$ & $\min$ & $\max$ & $\min$ & $\max$ \\
\hline 2D & & & 1 & 10 & 10 & 90 \\
\hline $3 \mathrm{D}$ & -1.5 & 1.5 & 1 & 10 & 5 & 90 \\
\hline
\end{tabular}
and in the $\beta \gamma$ planes.

TABLE I: Bounds of the input parameters.

a) 2D-example: the predicted inverse-mapped Voronoi cells $\hat{\Psi}_{i}$ in Fig. 2 and the noise levels $\hat{\Phi}_{i}^{\delta}$ for three different $\delta$ in Fig. 3 are shown $(n=25)$. Let us note that $\mathbb{D}_{25}$ is quasi output-equidistant, even so the shape and the dimensions of the different cells $\hat{\Psi}_{i}$ in the input-space are very diverse and illustrate the behavior of $\mathcal{F}$ in the different regions of $\mathbb{X}$. The large cells in the region of small cracks highlight the difficulties of inverting for such a kind of defects using our measurement configuration. On the contrary, the small cells in the region of large cracks show that such defects can be characterized precisely.

b) 3D-example: the predicted inverse-mapped noise cells $\hat{\Phi}_{i}^{\delta}$ are now depicted (Fig. 4, $n=216$ ). Only two different levels are drawn for three samples. This configuration is an extension of the previous 2D case and, as expected, the distribution of the input samples leads to the same conclusion for the two common parameters $L$ and $D$ : a quite homogeneous and fine sampling in the $L-A$ surface for high values of $D$ and a sparse sampling for low $D$ values. The inverse mapping shows a quite small volume around the points belonging to a dense region of $\mathbb{D}_{216}$ whereas a large volume is related to the samples in a sparse region. 


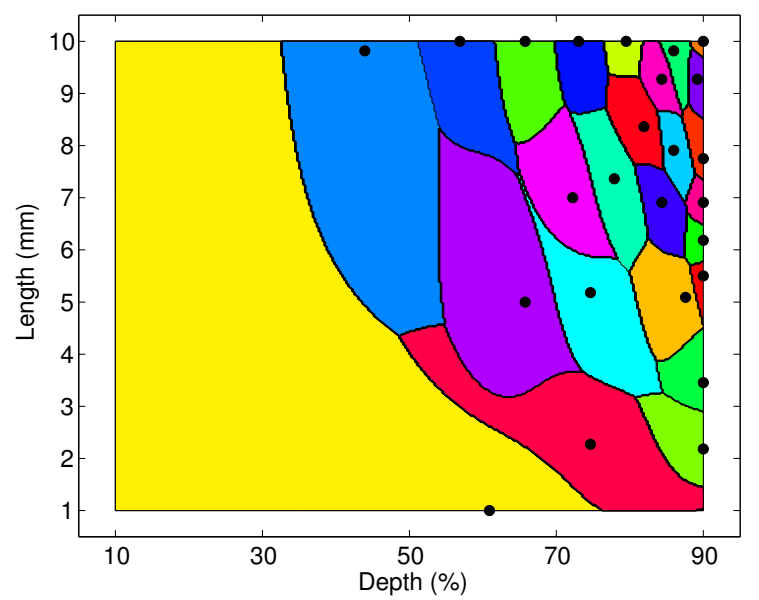

Fig. 2: 2D: inverse mapping of the Voronoi cells (dots: input samples).

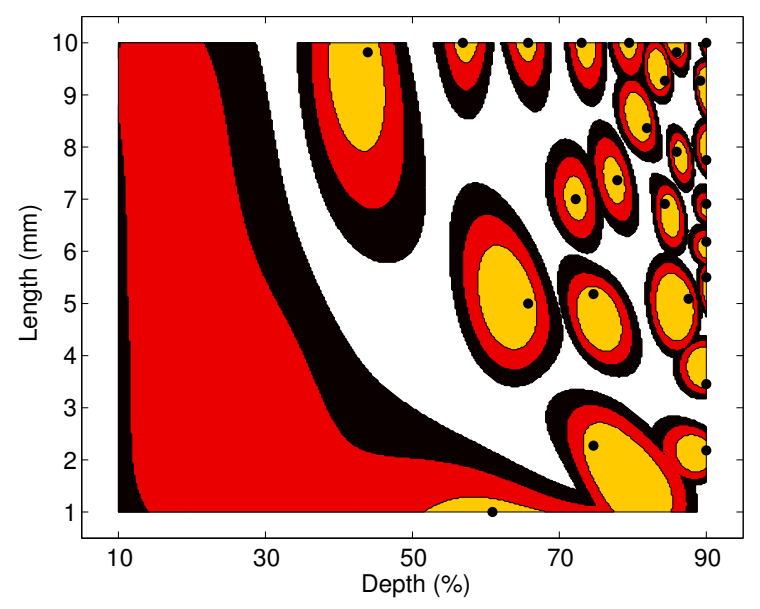

Fig. 3: 2D: inverse mapping of the noise cells for three different $\delta$ (dots: input samples).

\section{Conclusion}

The optimal database links an input space to an output space and it is built such that the latter is evenly filled by samples. The generation of the database is recast as an optimization problem, which is solved iteratively, by inserting the next input sample with respect to the distance between points in the output space. This is an expensive-to-compute optimization problem, thus, the use of a kriging-based interpolator is proposed.

The structure of the databases yields meta-information about the underlying problem to be emulated. Two inverse mappings are then proposed, one is related to the Voronoi cell mapping, the second links to a noise cell mapping. Both contribute to quantify the attainable precision in the solution of the corresponding inverse problem.

Such an approach can be extended to some other physical problems and so, its application to the characterisation of forest using radar observation data is under development.

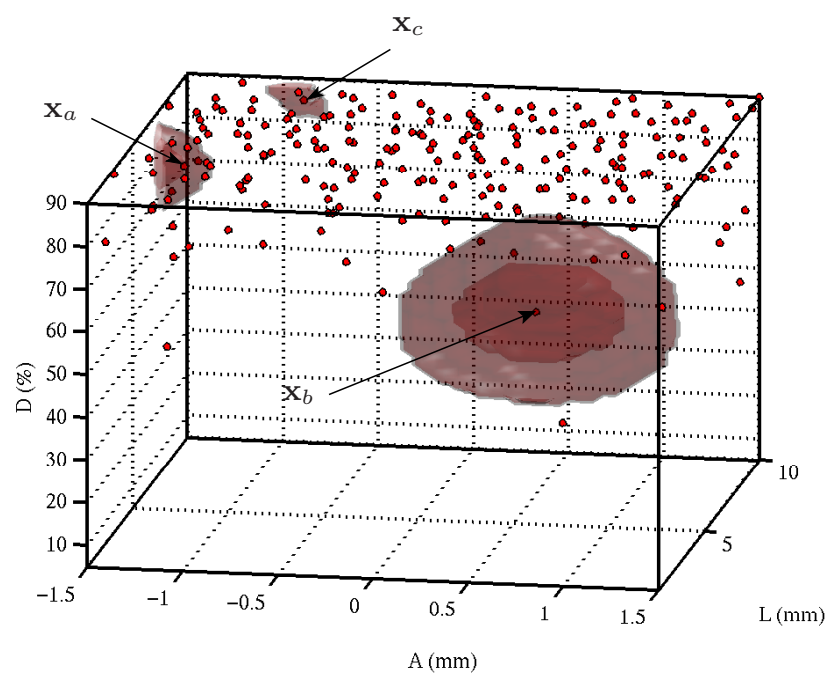

Fig. 4: 3D: inverse mapping of $\hat{\Phi}_{i}^{\delta}$ for two different $\delta . \mathbf{x}_{a}=$ $[-1.48 \mathrm{~mm} ; 8.34 \mathrm{~mm} ; 68.1 \%], \mathbf{x}_{b}=[0.56 \mathrm{~mm} ; 6.12 \mathrm{~mm}$; $51.2 \%], \mathbf{x}_{c}=[-0.86 \mathrm{~mm} ; 9.59 \mathrm{~mm} ; 86.4 \%]$ characterized by $[A ; L ; D]$ (dots: input samples).

\section{ACKNOWLEDGMENT}

The research is partially supported by the Research and Technology Innovation Fund of the Hungarian Government in the frame of the French-Hungarian Bilateral Intergovernmental S\&T Cooperation (FR-1/2008) and by DIGITEO cluster's project.

\section{REFERENCES}

[1] J. K. Sykulski, "New trends in optimization in electromagnetics," Przeglad Elektrotechniczny, vol. 83, no. 6, pp. 13-18, 2007.

[2] J. Pávó and S. Gyimóthy, "Adaptive inversion database for electromagnetic nondestructive evaluation," NDT\&E International, vol. 40, no. 3, pp. 192-202, 2007.

[3] S. Gyimóthy, I. Kiss, and J. Pávó, "Adaptive sampling technique based on moving meshes for building data-equidistant inversion databases for NDT," International Journal of Applied Electromagnetics and Mechanics, vol. 30, no. 3-4, pp. 309-319, 2009.

[4] R. Bettinger, P. Duchêne, L. Pronzato, and E. Thierry, "Design of experiments for response diversity," Journal of Physics: Conference Series, vol. 135, p. 012017, 2008.

[5] S. Bilicz, M. Lambert, E. Vazquez, and S. Gyimóthy, "A new database generation method combining maximin method and kriging prediction for eddy-current testing," in Proceedings of 14th International Workshop on Electromagnetic Nondestructive Evaluation, Dayton, USA, 2009, pp. 84-85.

[6] _ "A new database generation method combining maximin method and kriging prediction for eddy-current testing," in Electromagnetic NonDestructive Evaluation (XIII), ser. Studies in Applied Electromagnetics and Mechanics. Amsterdam: IOS Press, 2010, to appear, 8 pages.

[7] J. Chilès and P. Delfiner, Geostatistics, Modeling Spatial Uncertainty. Wiley, 1999.

[8] J. P. Kleijnen, "Kriging metamodeling in simulation: A review," European Journal of Operational Research, vol. 192, no. 3, pp. 707-716, February 2009.

[9] J. Pávó and D. Lesselier, "Calculation of eddy current testing probe signal with global approximation," IEEE Transactions on Magnetics, vol. 42, pp. 1419-1422, April 2006 